\title{
GROUP ACTIONS ON SPHERE BUNDLES OVER SPHERES
}

\author{
By Hiromichi Matsunaga
}

\section{Introduction.}

In a previous paper [8], we studied group actions on $S^{k}$-bundles over $S^{n}$ in cases of (1) $k<n \leqq 8$, (2) $k \geqq n$ and (3) $k=n-1, n \equiv 0 \bmod 4$. It is the purpose of this paper to estimate degrees of symmetry of bundle spaces and to construct group actions on semistable bundles over spheres.

Using Ku-Mann-Sicks-Su's theorems [5], [6], we shall give some estimation for upper bounds on degrees of symmetry in $\S 1$. In $\S 2$, we shall construct generators of stable groups $\pi_{4 s-1}(S O)$ which yield group actions on bundle spaces. Some theorems due to Kervaire [4] provide an information on generators of semistable groups $\pi_{i}(S O(n))$. In $\S 3$ we shall obtain some group actions on semistable sphere bundles over spheres. I would like to thank Professor S. Sasao for helpful conversations.

\section{$\S 1$. Degree of symmetry.}

For a closed connected smooth manifold $M$, the degree of symmetry of $M$ denoted by $N(M)$ is defined as the upper bound of the dimensions of all compact Lie groups which act effectively and smoothly on $M$. Then we have next propositions.

Proposition 1. Let $B$ be an $S^{k}$-bundle over $S^{n}$, where $n \geqq 9$. Then $B$ can not be a homotopy sphere.

Proof. If $B$ is a sphere, then by 28.2 and 28.6 in [9], we have $k=n-1$, and $n=1,2$ or $n \equiv 0 \bmod 4$, where the key point is that a fibre $S^{k}$ is contractible to a point in $B$, then we can replace the standard sphere in [9] by a homotopy sphere. Since $n=4 s$ and $s \geqq 3$, the space $B$ has a cell complex structure $B=$ $S^{n-1} \cup_{2 m \ell_{n-1}} \cup e^{n} \cup e^{2 n-1}$ (cf. 3 in [7]), which is a contradiction.

Proposition 2. Suppose that an $S^{k}$-bundle over $S^{n}$ admits a cross section or $k<n-1$, then we have

$$
N(B) \leqq \frac{1}{2} n(n+1)+\frac{1}{2} k(k+1) \quad \text { for } n+k \geqq 19 .
$$

Received January 8, 1982 
Proof. If the bundle $B$ admits a cross section or $k<n-1$, then the cohomology group $H^{n}(B, Q)$ with rational coefficient is non zero. Hence by Theorem 1 in [5] we obtain the proposition.

Proposition 3. Let $B$ be an $S^{n-1}$-bundle over $S^{n}$, where $n \geqq 10$. Suppose that $B$ is not homotopically equivalent to the product $S^{n-1} \times S^{n}$, then we have

$$
N(B) \leqq n^{2}-1 \text {. }
$$

Proof. Suppose that $N(B)>n^{2}-1$, then $N(B)>(1 / 4)(2 n-1)^{2}+(1 / 2)(2 n-1)$. By theorem 2 in [6], $B=\partial\left(D^{k} \times X\right), k \geqq n+1$, where $D^{k}$ denotes a $k$-disk and $X$ is a compact manifold with possibly boundary.

Case of $\partial X \neq \emptyset$.

Consider the homology exact sequence of the pair $\left(D^{k} \times X, B\right)$ :

$$
\longrightarrow H_{\imath+1}\left(D^{k} \times X\right) \longrightarrow H_{\imath+1}\left(D^{k} \times X, B\right) \longrightarrow H_{i}(B) \longrightarrow H_{\imath}\left(D^{k} \times X\right) \longrightarrow .
$$

We have isomorphisms $H_{\imath+1}\left(D^{k} \times X, B\right) \approx H_{\imath+1}\left(S^{k} \wedge(X / \partial X)\right) \approx H_{\imath-k+1}(X / \partial X)$ and $H_{i}\left(D^{k} \times X\right) \approx H_{\imath}(X)$. When $\imath=n-1$, we have an exact sequence

$$
\longrightarrow H_{n-k}(X / \partial X) \longrightarrow H_{n-1}(B) \longrightarrow H_{n-1}(X) \longrightarrow H_{n-k-1}(X / \partial X) \longrightarrow .
$$

Since the dimension of the manifold $X$ is less than or equal to $n-1$, we have $H_{n-1}(X)=0$, and $H_{n-k}(X / \partial X) \approx H_{n-k-1}(X / \partial X)=0$, because $k \geqq n+1$. Thus $H_{n-1}(B)$ $=0$. On the other hand, if $B$ yields a cross section then $H_{n-1}(B) \approx Z$, and by the proposition $1, B$ can not be a sphere, hence $H_{n-1}(B) \neq 0$ if $B$ does not yield any cross section. Thus any way we have $H_{n-1}(B) \neq 0$, which is a contradiction.

Case of $\partial X=\emptyset$.

In this case $B=S^{k-1} \times X$ and $k-1 \geqq n$. By the proposition $1, B$ can not be a sphere, then $X$ is a positive dimensional manifold. Thus $k-1=n$ and $X$ is an $n-1$ dimensional manifold. We have $H_{n-1}(X) \approx Z$. Since $H_{\imath}(B)=0$ for $0<\imath<n-1$, by Künneth formula, $H_{\imath}(X)=0$ for $0<\imath<n-1$. Hence $X$ is a homology sphere. Since $X$ is simply connected it is a homotopy sphere, which contradicts the assumption.

\section{$\S 2$. Stable bundles.}

In the previous paper [8], we studied group actions on $S^{k}$-bundles over $S^{n}$ for $k \geqq n$. Now using Barratt-Mahowald's formula, we can construct large group actions on these bundle spaces

Proposition 4. Any $S^{q+1}$-bundle over $S^{q+1}$ admits an $S O((1 / 2)(q+1))$-action if $q$ is odd and an $S O((1 / 2) q)$-action if $q$ is even, where $q \geqq 23$.

Proof. By Theorem 2 in [2], the homomorphism $\pi_{q}(S O(n)) \rightarrow \pi_{q}(S O(q+2))$ is epimorphic if $q \leqq 2(n-1)-1$ and $n \geqq 13$, where the homomorphism is the one 
induced by the inclusion map $S O(n) \subset S O(q+2)$. The structure group of the bundle can be reduced to $S O((1 / 2)(q+3))$ if $q$ is odd and $S O((1 / 2) q+2)$ if $q$ is even and $q \geqq 23$. By the inclusion maps

$$
\begin{array}{ll}
S O\left(\frac{1}{2}(q+3)\right) \times S O\left(\frac{1}{2}(q+1)\right) \subset S O(q+2) & \text { for odd } q, \\
S O\left(\frac{1}{2} q+1\right) \times S O\left(\frac{1}{2} q\right) \subset S O(q+2) & \text { for even } q,
\end{array}
$$

we have required actions.

Next we consider stable sphere bundles over $S^{4 k}$ for $k \geqq 3$. Here we shall take an analogy of [1] with a view to construct actions.

Let $\varepsilon_{7}: S^{7} \rightarrow S O(8)$ be the map defined by $\varepsilon_{7}(x)(y)=x y$ for $x, y \in S^{7}$, where the multiplication in $S^{7}$ is that of Cayley numbers. Further, let $\varepsilon_{k-1}: S^{k-1} \rightarrow$ $S O(k), k \equiv 1,2,4 \bmod 8$ be the map which gives a respresentative of a generator of the stable group $\pi_{k-1}(S O)$ by the inclusion map $S O(k) \rightarrow S O$. Clearly the bundle $S^{k-1} \rightarrow B \rightarrow S^{k}$ corresponding to $\varepsilon_{k-1}$ admits an $S^{1}$-action for $k=1,2$, an $S^{3}$-action for $k=4$ and a $G_{2}$-action for $k=8$ [8]. The map $\varepsilon_{k-1}$ defines a complex over the $k$-disk $D^{k}$,

$$
E\left(\varepsilon_{k-1}\right): 0 \longrightarrow D_{1}^{k} \times R^{k} \stackrel{\varepsilon_{k-1}}{\longrightarrow} D_{2}^{k} \times R^{k} \longrightarrow 0 \quad \text { (cf. Lemma } 10.1 \text { in [1]). }
$$

Denote by $\varepsilon_{k-1}^{*}: S^{k-1} \rightarrow S O(k)$ the map given by $\varepsilon_{k-1}^{*}(x)=$ the transposed matrix of $\varepsilon_{k-1}(x)$ for $x \in S^{k-1}$. Let

$$
E\left(\tilde{\varepsilon}_{8+k-1}\right): 0 \longrightarrow F_{1} \longrightarrow F_{0} \longrightarrow 0,
$$

be the complex over $D^{8} \times D^{k}$, which is defined by

$$
F_{1}=D^{8} \times D^{k} \times\left(R^{8} \otimes R^{k} \oplus R^{8} \otimes R^{k}\right), \quad F_{0}=D^{8} \times D^{k} \times\left(R^{8} \otimes R^{k} \oplus R^{8} \otimes R^{k}\right)
$$

and

$$
\begin{gathered}
\tilde{\varepsilon}_{8+k-1}\left(x_{1}, x_{2}\right)=\frac{1}{2}\left(\begin{array}{cc}
1 \otimes \varepsilon_{k-1}\left(x_{2}\right) & \varepsilon_{7}\left(x_{1}\right) \otimes 1 \\
\varepsilon_{7}^{*}\left(x_{1}\right) \otimes 1 & -1 \otimes \varepsilon_{k-1}^{*}\left(x_{2}\right)
\end{array}\right): S^{8+k-1} \longrightarrow S O(16 k), \\
\quad \text { for }\left(x_{1}, x_{2}\right) \in D^{8} \times S^{k-1} \cup S^{7} \times D^{k}=S^{8+k-1} .
\end{gathered}
$$

By (10.4) in [1], we have $\chi\left(E\left(\tilde{\varepsilon}_{8+k-1}\right)\right)=\chi\left(E\left(\varepsilon_{7}\right)\right) \cdot \chi\left(E\left(\varepsilon_{k-1}\right)\right)$, where $\chi$ is the Euler characteristic of a complex. Due to Bott periodicity $\chi\left(E\left(\tilde{\varepsilon}_{8+k-1}\right)\right)$ gives a generator of the group $K O\left(S^{8+k}\right)$ and determines an $S^{16 k-1}$-bundle over $S^{8+k}$, say $B^{(8+k, 16 k-1)}$. Then we have

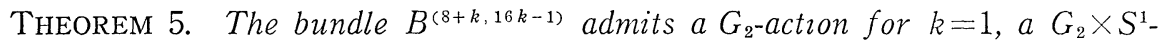
action for $k=2$, $a G_{2} \times S^{3}$-action for $k=4$ and $a G_{2} \times G_{2}$-action for $k=8$.

Proof. Consider the case $k=4$. For $\left(x_{1}, x_{2}\right) \in S^{7} \times S^{3},\left(y_{0} \otimes y_{1}^{\prime} \oplus y_{1} \otimes y_{0}^{\prime}\right) \in$ $R^{8} \otimes R^{4} \oplus R^{8} R^{4}$, we define an action of $(g, q) \in G_{2} \times S^{3}$ by 


$$
\begin{aligned}
& (g, q)\left\{\left(x_{1}, x_{2}\right),\left(y_{0} \otimes y_{1}^{\prime} \oplus y_{1} \otimes y_{0}^{\prime}\right)\right\} \\
= & \left\{\left(g\left(x_{1}\right), q x_{2} q^{-1}\right),\left(g\left(y_{0}\right) \otimes q y_{1}^{\prime} q^{-1} \oplus g\left(y_{1}\right) \otimes q y_{0}^{\prime} q^{-1}\right)\right\},
\end{aligned}
$$

then the clutching map $\tilde{\varepsilon}_{11}$ is compatible with the action (cf. $\S 1$ in [8]). Remainder cases $k=1,2$ and 8 are treated similarly.

COROLLARY 6. The generator of $\pi_{8 l+k-1}\left(S O\left(16^{l} \cdot k\right)\right)$ gives a stable sphere bundle admitting a $\left(G_{2}\right)^{l}$-action for $k=1, a\left(G_{2}\right)^{l} \times S^{1}$-action for $k=2, a\left(G_{2}\right)^{l} \times$ $S^{3}$-action for $k=4$ and $a\left(G_{2}\right)^{l+1}$-action for $k=8$.

Proof. These generators are given inductively by the map

$$
\frac{1}{2}\left(\begin{array}{cl}
1 \otimes \tilde{\varepsilon}_{8(l-1)+k-1} & \varepsilon_{7} \otimes 1 \\
\varepsilon_{7}^{*} \otimes 1 & -1 \otimes \tilde{\varepsilon}_{8(l-1)+k-1}^{*}
\end{array}\right): S^{8 l+k-1} \longrightarrow S O\left(16^{l} \cdot k\right) .
$$

Then we can construct desired actions analogously as the theorem.

\section{$\S 3$. Semistable bundles.}

The group structure of $\pi_{8 s+r}(S O(8 s+r-k))$ has given by the table in [4] for $s \geqq 1$ and $-1 \leqq k \leqq 4$. Here we shall give generators of these groups in order to obtain some information for the proof of the existence of group actions. We use the surjectivity of $j_{*}: \pi_{i}(S O(n)) \rightarrow \pi_{i}(S O)$ by Barratt-Mahowald, then the restriction $s \geqq 4$ is required. In order to obtain generators, we shall depend on the followings:

(1) the homotopy exact sequence associated to the fibering $S O(n-1) \rightarrow S O(n)$ $\rightarrow S^{n-1}$,

(2) boundary formulas of Theorems 1, 2 and 3, and Lemma 1 in [4]. Relations for generators of stable groups given by Lemma 2 in [4],

(3) the properties of characteristic maps given by 23.4, 24.3 and 24.5 in [9], and the structure of tangent bundles which is given by $27.8-27.11$ in [9],

(4) the distributive law in homotopy groups of spheres,

$$
\left(\beta_{1}+\beta_{2}\right) \circ(E \alpha)=\beta_{1} \circ(E \alpha)+\beta_{2} \circ(E \alpha) .
$$

Let $j^{(n, n-l)}: S O(n-l) \rightarrow S O(n)$ be the inclusion map. If $x \in \pi_{j}(S O(n))$ yields $y \in \pi_{i}(S O(n-l))$ such that $j_{*}^{(n, n-l)}(y)=x$, then we use a symbol $x^{(-l)}$ for $y$. Further we use the following symbols:

$\tau_{n-1}$; the characteristic map of the tangent bundle of $S^{n}$,

$\varepsilon_{4 s-1}$; one of the generators of $\pi_{4 s-1}(S O(4 s))$ such that $j_{*}^{(4 s+1,4 s)}\left(\varepsilon_{4 s-1}\right)$ gives the generator of the stable group $\pi_{4 s-1}(S O(4 s+1))$,

$\eta_{n}$; the generator of the stable group $\pi_{n+1}\left(S^{n}\right)$,

$\theta_{n}$; the generator of the stable group $\pi_{n+2}\left(S^{n}\right)$,

$\nu_{n}$; the generator of the stable group $\pi_{n+3}\left(S^{n}\right)$.

Now the generators of $\pi_{8 s+r}(S O(8 s+r-k))$, for $-1 \leqq k \leqq 4,0 \leqq r \leqq 7, s \geqq 4$ are given by the following table (1). 
Table (1)

\begin{tabular}{|c|c|c|c|c|c|}
\hline \multicolumn{2}{|c|}{$r \backslash k$} & \multirow{3}{*}{$\begin{array}{l}-1 \\
+Z_{2} \\
\varepsilon_{8 s-1} \eta_{8 s-1} \\
+Z_{2} \\
\varepsilon_{8 s-1} \theta_{8 s-1}\end{array}$} & \multicolumn{2}{|r|}{0} & \multirow[b]{2}{*}{$\varepsilon_{8 s-1}^{(-2)} \eta_{8 s-1},\left(\tau_{8 s-1} \eta_{8 s-1}\right)^{(-1)}$} \\
\hline 0 & $\begin{array}{l}Z_{2} \\
\tau_{8 s}\end{array}$ & & $\begin{array}{l}Z_{2} \\
\tau_{8 s}^{(-1)}, \varepsilon_{8 s-1}^{(-1)}\end{array}$ & $\begin{array}{lr}+Z_{2} & +Z_{2} \\
\eta_{8 s-1}, \tau_{8 s-1} \eta_{8 s-1}\end{array}$ & \\
\hline 1 & $\begin{array}{l}Z \\
\tau_{8 s+1}\end{array}$ & & $\begin{array}{l}Z_{2} \\
\varepsilon_{8 s-1}^{(-1)} \theta_{8 s-1}\end{array}$ & $\begin{array}{l}+Z_{2} \\
\tau_{8 s} \eta_{8 s}\end{array}$ & $\begin{array}{lcc}Z_{2} & +Z_{2} & +Z_{2} \\
\tau_{8 s-1} \theta_{8 s-1}, & \tau_{8 s}^{(-1)} \eta_{8 s}, \varepsilon_{8 s-1}^{(-2)} \theta_{8 s-1}\end{array}$ \\
\hline 2 & $\begin{array}{l}Z_{2} \\
\tau_{8 s+2}\end{array}$ & & $\begin{array}{l}Z_{4} \\
\tau_{8+2}^{(-1)}\end{array}$ & & $\begin{array}{l}Z_{8} \\
\tau_{8 s+2}^{(-2)}\end{array}$ \\
\hline 3 & $\begin{array}{l}Z \\
\varepsilon_{8 s+3}\end{array}$ & $\begin{array}{l}+Z \\
\tau_{8 s+3}\end{array}$ & $\begin{array}{l}Z \\
\varepsilon_{8 s+3}^{(-1)}\end{array}$ & & $\begin{array}{l}Z \\
\varepsilon_{8 s+3}^{(-2)}\end{array}$ \\
\hline 4 & $\begin{array}{l}Z_{2} \\
\tau_{8 s+4}\end{array}$ & & $\begin{array}{l}Z_{2} \\
\tau_{8 s+4}^{(-1)}\end{array}$ & $\begin{array}{l}+Z_{2} \\
\tau_{8 s+3} \eta_{8 s+3}\end{array}$ & $\begin{array}{l}Z_{2} \\
\left(\tau_{8 s+3} \eta_{8 s+3}\right)^{(-1)}\end{array}$ \\
\hline 5 & $\begin{array}{l}Z \\
\tau_{8 s+5}\end{array}$ & & $\begin{array}{l}Z_{2} \\
\tau_{8 s+4} \eta_{8 s+4}\end{array}$ & & $\begin{array}{ll}Z_{2} & +Z_{2} \\
\tau_{8 s+3} \theta_{8 s+3}, & \tau_{8 s+4}^{(-1)} \eta_{8 s+4}\end{array}$ \\
\hline 6 & $\begin{array}{l}Z_{2} \\
\tau_{8 s+6}\end{array}$ & & $\begin{array}{l}Z_{4} \\
\tau_{8 s+6}^{(-1)}\end{array}$ & & $\begin{array}{l}Z_{8} \\
\tau_{8 s+6}^{(-2)}\end{array}$ \\
\hline 7 & $\begin{array}{l}Z \\
\varepsilon_{8 s+7},\end{array}$ & $\begin{array}{c}+Z \\
\tau_{8 s+7}\end{array}$ & $\begin{array}{l}Z \\
\varepsilon_{8 s+7}^{(-1)}\end{array}$ & & $\begin{array}{l}Z \\
\varepsilon_{8 s+7}^{(-2)}\end{array}$ \\
\hline
\end{tabular}

Table (1)

\begin{tabular}{|c|c|c|c|c|c|c|}
\hline \multirow{2}{*}{$\frac{r \backslash k}{0}$} & \multicolumn{2}{|l|}{2} & \multicolumn{2}{|l|}{3} & \multicolumn{2}{|c|}{4} \\
\hline & $\begin{array}{l}Z_{12} \\
\tau_{8 s-3} \nu_{8 s-3}\end{array}$ & $\begin{array}{l}+Z_{2} \\
\varepsilon_{8 s-1}^{(-3)} \eta_{8 s-1}\end{array}$ & $\begin{array}{l}Z_{2} \\
\varepsilon_{8 s-1}^{(-4)} \eta_{8 s-}\end{array}$ & & $\begin{array}{l}Z_{2} \\
\varepsilon_{8 s-1}^{(-5)} \eta_{8 s}\end{array}$ & \\
\hline 1 & $\begin{array}{l}Z_{2} \\
\left(\tau_{8 s-1} \theta_{8 s-1}\right)^{(-1)}\end{array}$ & $\begin{array}{l}+Z_{2} \\
\varepsilon_{8 s-1}^{(-3)} \theta_{8 s-1}\end{array}$ & $\begin{array}{l}Z_{2} \\
\varepsilon_{8 s-1}^{(-4)} \theta_{8 s-}\end{array}$ & & $\begin{array}{l}Z_{2} \\
\varepsilon_{8 s-1}^{(-5)} \theta_{8 s}\end{array}$ & \\
\hline 2 & $\begin{array}{l}Z_{24} \\
\tau_{8 s-1} \nu_{8 s-1}\end{array}$ & $\begin{array}{l}+Z_{8} \\
\tau_{8 s+2}^{(-3)}\end{array}$ & $\begin{array}{l}Z_{8} \\
a\end{array}$ & & $\begin{array}{l}Z_{8} \\
a^{(-1)}\end{array}$ & \\
\hline 3 & $\begin{array}{l}Z \\
\varepsilon_{8 s+3}^{(-3)}\end{array}$ & $\begin{array}{c}+Z_{2} \\
\tau_{8 s} \nu_{8 s}\end{array}$ & $\begin{array}{l}Z \\
\varepsilon_{8 s+3}^{(-4)}\end{array}$ & $\begin{array}{l}+Z_{2} \\
\left(\tau_{8 s}^{(-1)} \nu_{8 s}\right)\end{array}$ & $\begin{array}{l}Z \\
\varepsilon_{8 s+3}^{(-5)}\end{array}$ & $\begin{array}{l}+Z_{2} \\
\left(\tau_{8 s}^{(-1)} \nu_{8 s}\right)^{(-1)}\end{array}$ \\
\hline 4 & $\begin{array}{l}Z_{12} \\
\tau_{8 s+1} \nu_{8 s+1}\end{array}$ & & 0 & & 0 & \\
\hline 5 & $\begin{array}{l}Z_{2} \\
\tau_{8 s+2} \nu_{8 s+2} \quad\left(\tau_{8 s+}\right.\end{array}$ & $\begin{array}{l}+Z_{2} \\
\left.{ }_{3} \eta_{8 s+3}\right)^{(-1)} \eta_{8 s+4}\end{array}$ & $\begin{array}{l}Z_{2} \\
\left(\left(\tau_{8 s+3} \eta_{8}\right.\right.\end{array}$ & $\left.\left.{ }_{+3}\right)^{(-1)} \eta_{8 s+4}\right)^{(-1)}$ & $\begin{array}{l}Z_{2} \\
\left(\left(\tau_{8 s+3} \eta\right.\right.\end{array}$ & $\left.\left.{ }_{s s+3}\right)^{(-1)} \eta_{8 s+4}\right)^{(-2)}$ \\
\hline 6 & $\begin{array}{l}Z_{4} \\
(b)\end{array}$ & $\begin{array}{l}+Z_{24 d} \\
(c)\end{array}$ & $\begin{array}{l}Z_{8} \\
\tau_{8 s+6}^{(-4)}\end{array}$ & & $\begin{array}{l}Z_{8} \\
\tau_{8 s+6}^{(-5)}\end{array}$ & \\
\hline 7 & $\begin{array}{l}Z \\
\varepsilon_{8 s+7}^{(-3)}\end{array}$ & $\begin{array}{l}+Z_{2} \\
\tau_{8 s+4} \nu_{8 s+4}\end{array}$ & $\begin{array}{l}Z \\
\varepsilon_{8 s+7}^{(-4)},\end{array}$ & $\begin{array}{l}+Z_{2} \\
\tau_{8 s+4}^{(-1)} \nu_{8 s+4}\end{array}$ & $\begin{array}{l}Z \\
\varepsilon_{8 s+7}^{(-5)}\end{array}$ & $\begin{array}{l}+Z_{2} \\
\left(\tau_{8 s+4}^{(-1)} \nu_{8 s+4}\right)^{(-1)}\end{array}$ \\
\hline
\end{tabular}


We have some relations on these generators:

$$
\begin{aligned}
& \tau_{8 s}^{(-1)} \simeq T_{4 s+1}^{\prime}: S^{8 s} \longrightarrow U(4 s) \quad\left(( 5 ) \text { of } 2 4 . 2 \text { in } \left[9_{-\prime}^{-},\right.\right. \\
& \tau_{8 s+2}^{(-1)} \simeq T_{4 s+3}^{\prime}: S^{8 s+2} \longrightarrow U(4 s+1), \\
& \tau_{8 s^{\circ}} \theta_{8 s}=4 \tau_{8 s+2}^{(-2)}, \\
& \tau_{8 s+2}^{(-3)} \simeq T_{2 s+1}^{\prime \prime}: S^{8 s+2} \longrightarrow S p(2 s) \quad(24.11 \text { in }[9]), \\
& j_{*}^{(8 s, 8 s-1)}(a)=6 \tau_{8 s-1}{ }^{\circ} \nu_{8 s-1}+\tau_{8 s+2}^{(-3)}, \\
& \tau_{8 s}^{(-1)} \circ \nu_{8 s}=T_{4 s+1}^{\prime}{ }^{\circ} \nu_{8 s}: S^{8 s+4} \longrightarrow U(4 s), \\
& \tau_{8 s+4}^{(-1)} \simeq T_{4 s+5}^{\prime}: S^{8 s+4} \longrightarrow U(4 s+2), \\
& \tau_{8 s+4}^{\circ} \eta_{8 s+4} \simeq T_{4 s+5}^{\prime}{ }^{\circ} \eta_{8 s+4}: S^{8 s+5} \longrightarrow U(4 s+2), \\
& \tau_{8 s+6}^{(-1)} \simeq T_{4 s+7}^{\prime}: S^{8 s+6} \longrightarrow U(4 s+3), \\
& b=-\tau_{8 s+6}^{(-3)}+3 \tau_{8 s+3}{ }^{\circ} \nu_{8 s+3}, \quad c=\tau_{8 s+2}^{(-3)}-\tau_{8 s+3}{ }^{\circ} \nu_{8 s+3} \\
& \tau_{8 s+6}^{(-3)} \simeq T_{2 s+2}^{\prime \prime}: S^{8 s+6} \longrightarrow S p(2 s+1), \\
& \tau_{8 s+4}^{(-1)} \circ \nu_{8 s+4} \simeq T_{4 s+5}^{\prime} \nu_{8 s+4}: S^{8 s+7} \longrightarrow U(4 s+2) .
\end{aligned}
$$

Now we investigate group actions on a bundle space $D_{1}^{n} \times S^{k} \cup_{\gamma} D_{2}^{n} \times S^{k}$, where the action is to be compatible with the identification $(x, y) \equiv(x, \gamma(x) y)$ for $(x, y) \in S^{n-1} \times S^{k}$. We describe our results as a Table (2). In the table, each group denotes the one which is admitted by any bundle corresponding to a characteristic map in each block on the Table (1).

Proof of the results on the table (2).

We have a decomposition $\eta_{8 s-1}=\eta_{2} * 1\left(S^{8 s-4}\right): S^{8 s}=S^{3} * S^{8 s-4} \rightarrow S^{8 s-1}=S^{2} * S^{8 s-4}$, where $\eta_{2}$ is the Hopf map $S^{3} \rightarrow S^{2}$ and $1\left(S^{8 s-4}\right)$ denotes the identity map of $S^{8 s-4}$. $\eta_{2}$ is invariant under the principal $S^{1}$-action on $S^{3}$, then $\eta_{8 s-1}$ is also $S^{1}$-invariant. By Satz of 6.4 in [3] we have

$$
\tau_{8 s}(g x)(g y)=g \tau_{8 s}(x)(y) \quad \text { for }(x, y) \in S^{8 s} \times S^{8 s}, g \in S O(2) .
$$

Since $\varepsilon_{8 s-1} \circ \eta_{8 s-1}$ is homotopic to $\varepsilon_{8 s-1}^{(-2)} \circ \eta_{8 s-1}$ in $S O(8 s+1)$,

$$
\begin{aligned}
\varepsilon_{8 s-1} \circ \eta_{8 s-1}(g x) \tau_{8 s}(g x)(g y) & =\varepsilon_{8 s-1}^{(-2)} \circ \eta_{8 s-1}(x) g \tau_{8 s}(x)(y) \\
& =g \varepsilon_{8 s-1}^{(-2)} \circ \eta_{8 s-1}(x) \tau_{8 s}(x)(y) .
\end{aligned}
$$

Hence the bundle space with the characteristic map $\tau_{8 s}+\varepsilon_{8 s-1} \circ \eta_{8 s-1}$ admits an $S O(2)$-action. This is the result for $(r, k)=(0,-1)$.

Similar argument is valid for $(r, k)=(0,0),(0,1),(0,2),(1,-1),(1,0),(1,1)$.

By the surjectivity of the homomorphism $j_{*}: \pi_{8 s-1}(S O(4 s+1)) \rightarrow$ $\pi_{8 s-1}(S O(8 s-3))$, we have 


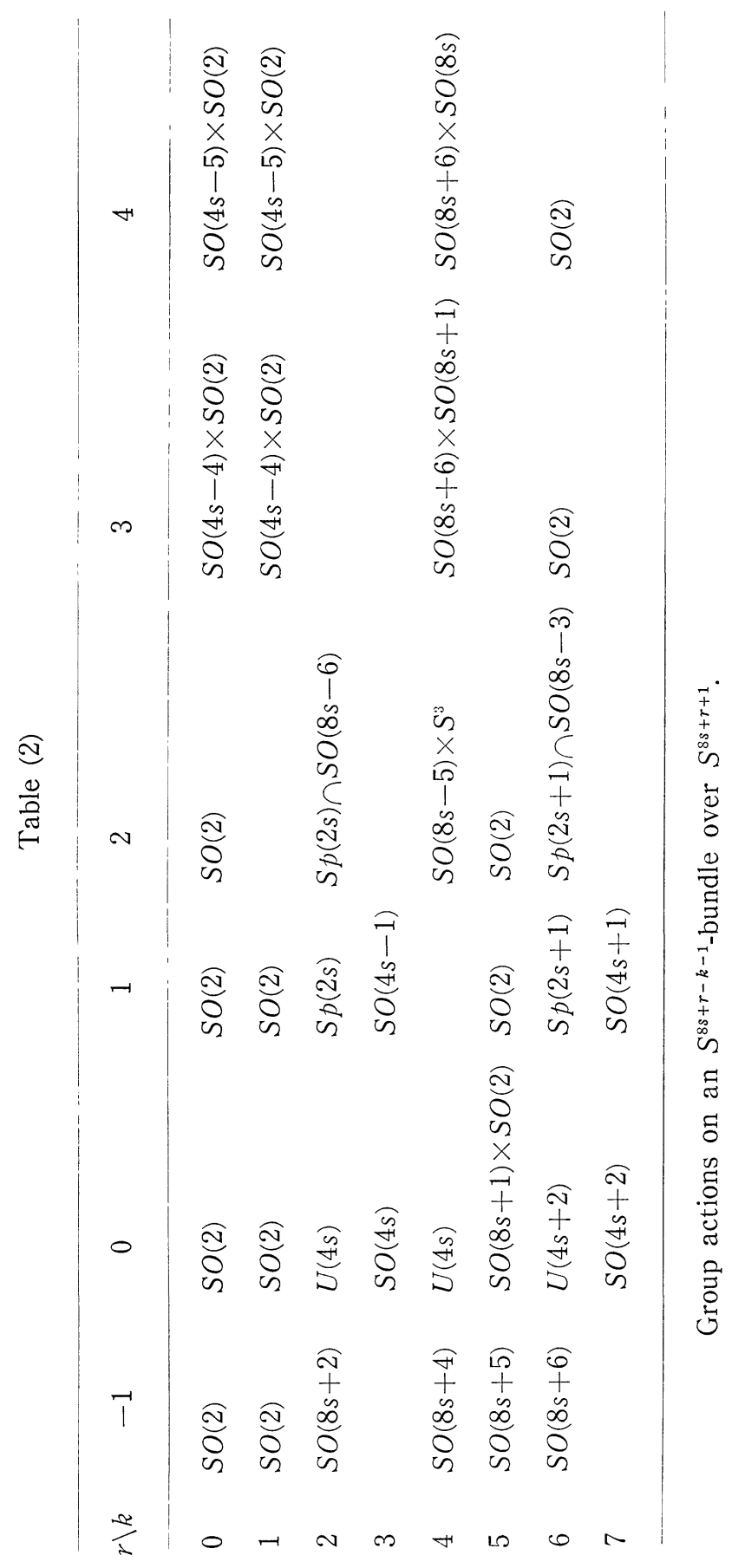




$$
\begin{aligned}
\varepsilon_{8 s-1}^{(-4)} \circ \eta_{8 s-1}\left(g_{1} x\right)\left(g_{2} y\right)=g_{2} \varepsilon_{8 s-1}^{(-4)} \eta_{8 s-1}(x)(y) \quad & \text { for }(x, y) \in S^{8 r} \times S^{8 r-4}, \\
& \left(g_{1}, g_{2}\right) \in S O(2) \times S O(4 s-4) .
\end{aligned}
$$

Then we have the result for $(r, k)=(0,3)$. Similar argument is valid for $(r, k)$ $=(0,4),(1,3),(1,4),(4,2)$.

Since we can obtain similar results for $\left\{T^{\prime}\right\}$ and $\left\{T^{\prime \prime}\right\}$ to Satz of 6.4 in [3], using the relations on generators, group actions are obtained in the cases of $(r, k)=(2,-1),(2,0),(2,1),(6,-1),(6,0),(6,1)$.

By the decomposition $\tau_{8 s-1} \circ \nu_{8 s-1}=\tau_{8 s-1} \circ\left(\nu_{4} * 1\left(S^{8 s-6}\right)\right)$, and the relation $\tau_{8 s+2}^{(-3)} \simeq$ $T_{2 s+1}^{\prime \prime}$, we have

$$
\begin{aligned}
& \tau_{8 s-1} \circ \nu_{8 s-1}(g x) T_{2 s+1}^{\prime \prime}(g x)(g y)=\tau_{8 s-1} \circ \mathcal{\nu}_{8 s-1}(g x)\left(g T_{2 s+1}^{\prime \prime}(x)(y)\right) \\
&=g \tau_{8 s-1} \circ \nu_{8 s-1}(x)(y), \\
& \text { for }(x, y) \in S^{8 s+2} \times S^{8 s-1}, g \in S p(2 s) \cap S O(8 s-6),
\end{aligned}
$$

where the action is given by $g\left(x_{1}, x_{2} ; t\right)=\left(x_{1}, g x_{2} ; t\right)$ for $x_{1} \in S^{7}, x_{2} \in S_{8}{ }^{s-6}$, $0 \leqq t \leqq 1$. Hence we have proved the cases of $(2,2)$ and similarly $(6,2)$.

Since $\tau_{8 s+6}^{(-7)} \neq 0$, we have group actions in the cases of $(6,3),(6,4)$.

We can obtain group actions for remainder cases. For empty blocks, I have not yet obtained group actions for general bundles (cf. $\S 3$ in [8]).

\section{REFERENCES}

[1] M.F. Atiyah, R. Bott and A. Shapiro, Clifford modules, Topology, 1. Suppl. 1 (1964), 3-38.

[2] M.G. Barratt and M. E. Mahowald, The meta stable homotopy of $O(n)$, Bull. Amer. Math. Soc. 70 (1964), 758-760.

[3] F. Hirzebruch ANd K.H. Mayer, $O(n)$-Mannigfaltigkeiten, exotische Sphären und Singularitäten, Springer, Lecture Notes in Math. 57 (1968).

[4] M. A. Kervaire, Some nonstable homotopy groups of Lie groups, Illinois J. Math. 4 (1960), 161-169.

[5] H.T. Ku, L. N. Mann, J.L. Sicks and J.C. Su, Degree of symmetry of a product manifolds, Tras. Amer. Math. Soc. 146 (1969), 133-149.

[6] H. T. Ku, L.N. MAnN, J. L. Sicks And J.C. Su, Degree of symmetry of closed manifolds, Springer, Lecture Notes in Math. 298 (1972), 430-436.

[7] H. Matsunaga, A note on the differentable structures of total spaces of sphere bundles over spheres, Osaka J. Math. 2 (1965), 63-69.

[8] H. Matsunaga, $S^{1}$-actions on sphere bundles over spheres, Mem. Fac. Sci. Shimane Univ. 15 (1981), 9-16.

[9] N. Steenrod, The topology of fibre bundles, Princeton, 1951.

Department of Mathematics,

SHIMANe University 